\title{
Burning Rate in Impinging Jet Flames
}

\author{
A. N. Lipatnikov \\ Department of Applied Mechanics, Chalmers University of Technology, 41296 Gothenburg, Sweden
}

Correspondence should be addressed to A. N. Lipatnikov, lipatn@chalmers.se

Received 14 February 2011; Accepted 11 April 2011

Academic Editor: Alexey A. Burluka

Copyright (๑) 2011 A. N. Lipatnikov. This is an open access article distributed under the Creative Commons Attribution License, which permits unrestricted use, distribution, and reproduction in any medium, provided the original work is properly cited.

A method for evaluating burning velocity in premixed turbulent flames stabilized in divergent mean flows is quantitatively validated using numerical approximations of measured axial profiles of the mean combustion progress variable, mean and conditioned axial velocities, and axial turbulent scalar flux, obtained by four research groups from seven different flames each stabilized in an impinging jet. The method is further substantiated by analyzing the combustion progress variable balance equation that is yielded by the extended Zimont model of premixed turbulent combustion. The consistency of the model with the aforementioned experimental data is also demonstrated.

\section{Introduction}

Although turbulent flame speed $S_{t}$, by analogy with laminar burning, is often considered to be a basic characteristic of premixed combustion and was the main subject of numerous studies reviewed elsewhere [1-4], a precise definition of this quantity is difficult due to the following two key differences between laminar and turbulent premixed flames.

First, while a typical laminar flame is thin as compared with both the flame curvature and scales of spatial nonuniformities of the incoming flow of fresh reactants, the mean thickness $\Delta_{t}$ of a turbulent flame is comparable with length scales that characterize mean flow nonuniformities or the flame curvature. Due to the significant thickness of a turbulent flame brush, both the mean convective flux of the deficient reactant toward the flame and the mean flow velocity vary substantially across the flame brush, thus making a choice of a reference flux or a reference flow velocity (with respect to that flame speed should be defined) difficult $[5,6]$. For instance, Figure 2 in a paper by Gouldin [6] indicates more than threefold decrease in the mean normal convective flux from the leading to the trailing edges of a $\mathrm{V}$-shaped flame brush. Other experimental data that show the same effect were recently reviewed by Lipatnikov and Chomiak [7] (see Section 5.2 in the cited paper). It is also worth remembering that the difference in the speeds of planar and curved laminar flames scales as a ratio $\delta_{L} / R_{f}$ of the flame thickness to the radius of its curvature [8], and this ratio is much less than unity in a typical laminar premixed flame. If a similar scaling is assumed to hold for a premixed turbulent flame, then the difference between the speeds of statistically planar and curved flames could be large [9], because a ratio of $\Delta_{t} / R_{f}$ is of unity order in a typical curved premixed turbulent flame.

Second, while a typical laminar flame has a fully developed structure and speed, because a time scale of laminar flame development is very short in comparison with time scales characterizing the flow, a typical turbulent flame is a developing wave, with the growth of $\Delta_{t}$ being the most striking manifestation of premixed turbulent flame development, as stressed elsewhere [3]. Due to the growth of a turbulent flame brush thickness, the observed speeds of different isotemperature (or isoconcentration) surfaces differ from one another.

In the statistically planar one-dimensional case, the latter problem may be resolved by considering turbulent burning velocity $U_{t} \equiv\left|\int_{-\infty}^{\infty} \bar{W} d x /\left\{\rho_{u}[Y(\infty)-Y(-\infty)]\right\}\right|$, that is, the normalized total mass rate of consumption (or production) of the deficient reactant (or a main product) $Y$ per unit mean flame area. However, in the case of a curved flame brush, the definition of the area is ambiguous due to a large thickness $\Delta_{t}$. For instance, a ratio of the areas of surfaces associated with the leading and trailing edges of a statistically spherical turbulent flame brush may be as large as $\left(R_{f}+\Delta_{t}\right)^{2} / R_{f}^{2} \approx$ 4 if the mean flame radius and thickness are of the same order. 
Several methods have been proposed to resolve the above issues and to determine turbulent flame speed $S_{t}$ and/or burning velocity $U_{t}$ in statistically stationary $[5,6]$ or expanding [10-12] flames. In particular, in [10], an expanding statistically spherical premixed turbulent flame with a self-similar mean structure (i.e., the mean density $\bar{\rho}(\xi)$ depends on a single normalized distance $\xi=[r-$ $\left.R_{f}(t)\right] / \Delta_{t}(t)$, rather than on two independent variables, time $t$ and radial distance $r$ ) was theoretically studied and the following two radii:

$$
\begin{gathered}
R_{f, s}=\left(3 \int_{0}^{\infty} \bar{c} r^{2} d r\right)\left(2 \int_{0}^{\infty} \bar{c} r d r\right)^{-1}, \\
R_{f, u}^{2}=2 \int_{0}^{\infty} \bar{c} d r
\end{gathered}
$$

were introduced. Here, $c$ is the well-known combustion progress variable [13] and overbar designates Reynolds average. It was analytically shown that (i) if turbulent burning velocity is defined as follows $U_{t} \equiv \int_{0}^{\infty} \bar{W} r^{2} d r /\left(\rho_{u} R_{f, u}^{2}\right)$, then it is not affected straightforwardly by the rate of the growth of the mean flame brush thickness, (ii) if turbulent flame speed is defined as follows $S_{t}=\left(\rho_{b} / \rho_{u}\right) d R_{f, s} / d t$, then $S_{t}=U_{t}$, and (iii) in order for the difference in the observed flame speed $d R_{f, s} / d t$ and a mean velocity $u_{u}$ of the unburned mixture to be equal to $S_{t}$ or $U_{t}$, the velocity $U_{u}$ should be evaluated by extrapolating the mean flow velocity distribution $\bar{u}(r)$ in the unburned mixture ahead of the flame brush to the burning velocity surface characterized by $r=R_{f, u}$. Here, $\bar{W}$ is the mean mass rate of product creation, that is, the source term in the well-known balance equation [13] for the Favreaveraged combustion progress variable.

It is of interest to note that, as shown elsewhere [12], the above purely theoretical results are very close to the definition of the burning velocity of expanding statistically spherical premixed turbulent flames, proposed by Bradley et al. [11] based on different reasoning.

Although the theoretical analysis in [10] was solely performed for expanding flames, either statistically planar or statistically spherical ones, it was also hypothesized that the proposed methods for determining $U_{t}, S_{t}$, and $U_{u}$ may be useful in other cases, for example, in statistically stationary premixed turbulent flames stabilized in divergent flows such as impinging jets. In particular, turbulent burning velocity in such a flame was hypothesized to be approximately equal to a velocity obtained by extrapolating the velocity distribution in the unburned gas ahead of the flame to a burning velocity surface characterized by the following reference value of the Favre-averaged combustion progress variable:

$$
c_{0}=\tilde{c}\left(\xi=\frac{\rho_{u}}{\rho_{b}} \int_{0}^{1} \Psi_{2} d \tilde{c}\right),
$$

where

$$
\rho_{u} \Psi_{2}(\xi) \equiv \bar{\rho} \xi-\int_{-\infty}^{\xi} \frac{d \bar{\rho}}{d \zeta} \zeta d \zeta,
$$

and $\xi=\left(x-x_{f}\right) / \Delta_{t}$ is normalized distance in the direction normal to the mean flame brush.

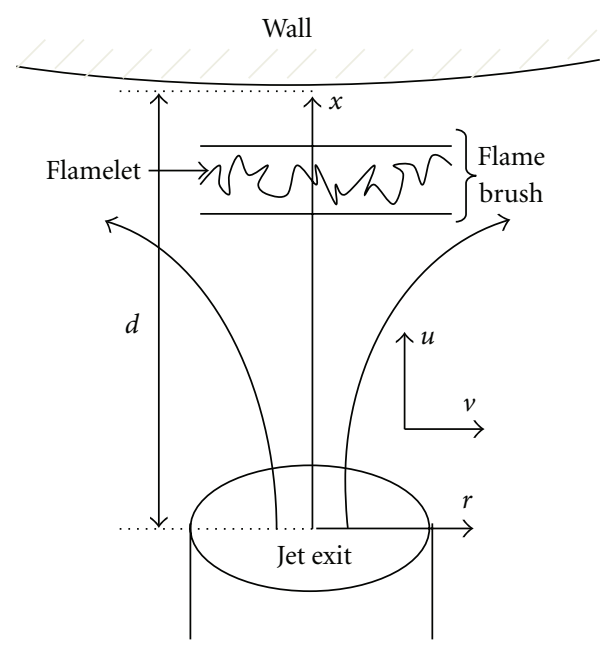

FIGURe 1: Premixed turbulent flame stabilized in an impinging jet.

In a subsequent paper [14], this hypothesis was indirectly supported by simulating a premixed turbulent flame stabilized in an impinging jet and by invoking two different RANS models of premixed turbulent combustion. However, in the cited paper, only numerical results were reported, and the discussed hypothesis has never been tested against experimental data, to the best of the author's knowledge.

The present work is aimed at filling this gap and testing the hypothesis against experimental data obtained by four research groups [15-18] from seven premixed turbulent flames each stabilized in an impinging jet.

In the next section, the hypothesis is tested. Obtained results are discussed in the third section followed by conclusions.

\section{Test}

Because the studied flames (see Figure 1 and Table 1) look statistically planar in the vicinity of the axis of an impinging jet, turbulent burning velocity may be evaluated by integrating the mean mass rate of product creation along the axis, that is,

$$
u_{t}=\int_{0}^{1} \Omega d z
$$

where the burning velocity $u_{t}=U_{t} / U$, burning rate $\Omega=$ $d \bar{W} / \rho_{u} U$, and axial distance $z=x / d(z=0$ at the wall) are normalized using the distance $d$ between the burner exit and the wall, the mean flow velocity $U$ at the burner exit, and the density $\rho_{u}$ of the unburned gas.

In the present work, the burning rate $\Omega$ is evaluated using the following well-known balance equation [19-24]:

$$
\begin{gathered}
\frac{d}{d z}(\bar{\rho} \tilde{w} \widetilde{c})+2 \bar{\rho} \widetilde{g} \widetilde{c}+\frac{d}{d z}\left[\bar{\rho} \widetilde{c}(1-\widetilde{c})\left(\bar{w}_{b}-\bar{w}_{u}\right)\right] \\
+2 \bar{\rho} \widetilde{c}(1-\widetilde{c})\left(\bar{g}_{b}-\bar{g}_{u}\right)=\Omega,
\end{gathered}
$$

which holds in the vicinity of the jet axis. Note that the radial turbulent scalar flux, that is, the last term on the left hand 
side (LHS) of (6), was taken into account in $[23,24]$, but was neglected in the other cited papers [19-22]. Here, we set $\rho_{u}=1$ for simplicity, that is, symbol $\rho$ designates the density normalized using the density of unburned gas, $\widetilde{w}=\tilde{u} / U$ is the normalized Favre-averaged axial velocity, which depend only on $z$ on the axis $(\partial \bar{c} / \partial r=\partial \bar{\rho} / \partial r=\partial \tilde{w} / \partial r=0$ at $r=0$ for symmetry reasons), and $g=(d / U)(\partial v / \partial r)_{r=0}$ is the normalized radial gradient of the radial velocity, calculated at the axis, with $\tilde{g}$ depending solely on $x$. The following wellknown BML relation [25]:

$$
\overline{\rho \mathbf{u}^{\prime \prime} c^{\prime \prime}}=\bar{\rho} \widetilde{c}(1-\tilde{c})\left(\overline{\mathbf{u}}_{b}-\overline{\mathbf{u}}_{u}\right)
$$

was invoked to close the turbulent scalar flux $\overline{\rho \mathbf{u}^{\prime \prime} c^{\prime \prime}}$ in the balance equation for $\tilde{c}$, with $\overline{\mathbf{u}}_{u}$ and $\overline{\mathbf{u}}_{b}$ designating velocity vectors conditioned to unburned and burned mixture, respectively.

In order to evaluate the normalized burning rate by solving (6), the axial distributions of $\widetilde{c}(z), \bar{\rho}(z), \widetilde{w}(z), \bar{w}_{u}(z)$, $\bar{w}_{b}(z), \bar{g}_{u}(z)$, and $\bar{g}_{b}(z)$ should be known. In the present work, the following well-known BML state equation [13]:

$$
\rho_{b} \bar{c}=\bar{\rho} \tilde{c}=\frac{\tilde{c}}{1+(\sigma-1) \tilde{c}}
$$

and the experimental data reported by Cho et al. [15], Cheng and Shepherd [16], Li et al. [17], and Stevens et al. [18] were used for these purposes. Here, $\sigma=\rho_{b}^{-1}$ is the density ratio. However, because the straightforward use of the experimental data could result in significant numerical errors when differentiating the data measured in a few points separated by substantial distances, smooth numerical approximations of these experimental data were obtained using the following method [24].

First, following the widely accepted practice [19-23], the axial profiles of either the Reynolds-averaged combustion progress variable, reported by Cho et al. [15] and by Cheng and Shepherd [16], or the Favre-averaged one, measured by Li et al. [17] and by Stevens et al. [18], were approximated (see Figure 2) using (i) a complementary error function

$$
\bar{c}=\frac{1}{2} \operatorname{erfc}(\sqrt{\pi} \xi)=\frac{1}{\sqrt{\pi}} \int_{\xi \sqrt{\pi}}^{\infty} \mathrm{e}^{\zeta^{2}} d \zeta
$$

to calculate $\bar{c}(z)$ and (ii) equation (8) to compute $\widetilde{c}[\bar{c}(z)]$. Here and in the following, $\xi=\left(z-z_{f}\right) / \delta_{t}$, with the values of the normalized flame coordinate $z_{f}$ and thickness $\delta_{t}=$ $\Delta_{t} / d$ being reported in Table 2 . As shown elsewhere [3, 27], this approximation works well in various premixed turbulent flames. The readers interested in a theoretical justification of (9) are referred to $[28,29]$.

Second, the profiles of the mean axial velocity and either axial conditioned velocities or axial turbulent scalar flux were computed by solving the following well-known [19-23] continuity:

$$
\frac{d \bar{\rho} \widetilde{w}}{d z}+2 \bar{\rho} \tilde{g}=0
$$

and radial impulse

$$
\begin{aligned}
& \frac{d}{d z}(\bar{\rho} \widetilde{w} \tilde{g})+3 \bar{\rho} \widetilde{g}^{2}+\frac{d}{d z}\left[\bar{\rho} \widetilde{c}(1-\widetilde{c})\left(\bar{w}_{b}-\bar{w}_{u}\right)\left(\bar{g}_{b}-\bar{g}_{u}\right)\right] \\
& +3 \bar{\rho} \widetilde{c}(1-\widetilde{c})\left(\bar{g}_{b}-\bar{g}_{u}\right)^{2}=Q,
\end{aligned}
$$

equations supplemented with (i) the following boundary conditions:

$$
\widetilde{w}(1)=-1, \quad \tilde{g}(1)=g_{1},
$$

(ii) the well-known BML relation [13]

$$
\overline{\mathbf{u}}=\widetilde{\mathbf{u}}+\overline{\mathbf{u}^{\prime \prime}}=\widetilde{\mathbf{u}}+(\sigma-1) \overline{\rho \mathbf{u}^{\prime \prime} c^{\prime \prime}},
$$

and (iii) balance equations for $\bar{g}_{u}$ and the difference in $\bar{w}_{b}$ and $\bar{w}_{u}$, derived by the present author [30] and discussed in detail elsewhere [7, 31]. Here, $Q=-d^{2} / U^{2}\left(\partial^{2} \bar{p} / \partial r^{2}\right)_{r=0}$ and $g_{1}$ are tuning parameters reported in Table 2 .

The readers interested in discussion and validation of this numerical model are referred to a recent paper [24]. For the goal of the present study, the validity of this model is a side issue, but the most important point consists of the fact that curves plotted in Figures 3, 4, and 5 well approximate experimental data shown in symbols. Therefore, these curves could be considered to be smooth approximations of the data, while further details of the approximation techniques are of minor importance as far as the evaluation of $\Omega$ by numerically integrating (6) is concerned.

Thus, in the present work, the reference method of evaluating turbulent burning velocity consists of numerically integrating (5) and (6) by invoking the smooth approximations of experimental data shown in lines in Figures 2, 3, 4 , and 5. The values of $u_{t}$ obtained using this method are reported in the first row of Table 3.

These reference values are used in order to test the method put forward in [10]. The latter method consists of the following.

First, the normalized velocity profile $\bar{w}(z)$ obtained in the unburned mixture is extrapolated by invoking the following analytical solution:

$$
\begin{gathered}
\bar{w}_{e}=2 g_{1}(1-z)-\left(g_{1}^{2}-Q\right)(1-z)^{2}-1, \\
\bar{g}_{e}=g_{1}-\left(g_{1}^{2}-Q\right)(1-z),
\end{gathered}
$$

to (10) and (11) written in the constant density case $(\rho=1)$ and supplemented with boundary conditions given by (12). In the present work, the parameters $Q$ and $g_{1}$ were taken from Table 2. If the method is applied to process raw experimental data, then these parameters may be tuned by comparing the profile of $\bar{w}(z)$ obtained from the oncoming flow of unburned mixture (e.g., see thin solid line in Figure 6) with profiles calculated using (14) with various $Q$ and $g_{1}$ (e.g., see dashed line in Figure 6).

Second, the reference value $c_{0}$ associated with the burning velocity surface is calculated using (3), (4), and (9). It can easily be shown that the reference surface is located at $z=z_{f}$ if (8) holds. Indeed, using (8) and (9), we have

$$
-\frac{\sigma}{\sigma-1} \frac{d \bar{\rho}}{d \xi}=\sigma \bar{\rho}^{2} \frac{d \tilde{c}}{d \xi}=\frac{d \bar{c}}{d \xi}=-\mathrm{e}^{-\pi \xi^{2}} .
$$


TABLe 1: Experimental conditions.

\begin{tabular}{lcclccccc}
\hline No. & $\begin{array}{c}d^{\mathrm{a}} \\
(\mathrm{m})\end{array}$ & $\begin{array}{c}U^{\mathrm{b}} \\
(\mathrm{m} / \mathrm{s})\end{array}$ & Fuel & $\Phi^{\mathrm{c}}$ & $\begin{array}{c}S_{L} \\
(\mathrm{~m} / \mathrm{s})\end{array}$ & $\sigma^{\mathrm{d}}$ & $\begin{array}{c}u^{\prime \mathrm{e}} \\
(\mathrm{m} / \mathrm{s})\end{array}$ & Flame \\
\hline 1 & 0.075 & 5 & $\mathrm{CH}_{4}$ & 1.0 & $0.365^{\mathrm{f}}$ & 7.513 & 0.30 & Cho et al. [15], case 1 \\
2 & 0.1 & $5^{\mathrm{g}}$ & $\mathrm{C}_{2} \mathrm{H}_{6}$ & 1.0 & $0.76^{\mathrm{h}}$ & 8.004 & 0.4 & Cheng and Shepherd [16], s9 \\
3 & 0.03 & 3.6 & $\mathrm{CH}_{4}$ & 0.89 & 0.307 & 7.077 & 0.2 & Li et al. [17], h4 ${ }^{\mathrm{i}}$ \\
4 & 0.03 & 3.6 & $\mathrm{CH}_{4}$ & 0.89 & 0.307 & 7.077 & 0.2 & Li et al. [17], h6 \\
5 & 0.035 & 0.75 & $\mathrm{CH}_{4}$ & 0.6 & 0.085 & 5.544 & 0.06 & Stevens et al. [18], set 1 \\
6 & 0.035 & 3 & $\mathrm{CH}_{4}$ & 1.0 & 0.365 & 7.513 & 0.33 & Stevens et al. [18], set 2 \\
7 & 0.035 & 2.25 & $\mathrm{CH}_{4}$ & 1.3 & 0.213 & 7.112 & 0.18 & Stevens et al. [18], set 3 \\
\hline
\end{tabular}

${ }^{\mathrm{a}} d$ is the distance between the jet exit and the wall (see Figure 1).

${ }^{\mathrm{b}} U$ is the mean axial flow velocity in the jet exit.

${ }^{\mathrm{c}} \Phi$ is the equivalence ratio.

$\mathrm{d}_{\sigma}$ is the density ratio.

${ }^{\mathrm{e}} u^{\prime}$ is the rms turbulent velocity.

${ }^{\mathrm{f}}$ For $\mathrm{CH}_{4}$-air mixtures, the values of the laminar flame speeds $S_{\mathrm{L}}$, reported in Table 1, were estimated using recent experimental data [26].

gThis value was reported by Bray et al. [19] (see footnote on page 645 in the cited paper).

${ }^{\mathrm{h}}$ This value was reported by Cheng and Shepherd [16].

${ }^{\mathrm{i}}$ The main difference between the two flames studied by Li et al. [17] was the diameters ( $h=4$ and $6 \mathrm{~mm}$ ) of holes in grids used to generate turbulence.

TABLE 2: Parameters of computations.

\begin{tabular}{lcccc}
\hline Flame & $z_{f}$ & $\delta_{t}$ & $Q$ & $g_{1}$ \\
\hline 1 & 0.217 & 0.080 & 1.298 & 0.074 \\
2 & 0.546 & 0.141 & 0.648 & 0.832 \\
3 & 0.154 & 0.062 & 1.733 & -0.183 \\
4 & 0.232 & 0.113 & 1.511 & -0.016 \\
5 & 0.362 & 0.112 & 0.899 & 0.398 \\
6 & 0.295 & 0.157 & 1.272 & 0.169 \\
7 & 0.259 & 0.116 & 1.215 & 0.155 \\
\hline
\end{tabular}

Subsequently, (4) and (15) read

$$
\begin{aligned}
\Psi_{2} & =\bar{\rho} \xi-\frac{\sigma-1}{\sigma} \int_{-\infty}^{\xi} \zeta \mathrm{e}^{-\pi \zeta^{2}} d \zeta \\
& =\bar{\rho} \xi+\frac{\sigma-1}{2 \pi \sigma} \mathrm{e}^{-\pi \xi^{2}} \\
& =\bar{\rho} \xi-\frac{\sigma-1}{2 \pi \sigma} \frac{d \bar{c}}{d \xi}
\end{aligned}
$$

Therefore,

$$
\begin{aligned}
\int_{0}^{1} \Psi_{2} d \tilde{c} & =\int_{\infty}^{-\infty}\left(\bar{\rho} \xi-\frac{\sigma-1}{2 \pi \sigma} \frac{d \bar{c}}{d \xi}\right) \frac{d \tilde{c}}{d \xi} d \xi \\
& =\int_{\infty}^{-\infty}\left(\frac{\xi}{\sigma \bar{\rho}} \frac{d \bar{c}}{d \xi}+\frac{1}{2 \pi \sigma \bar{\rho}^{2}} \frac{d \bar{\rho}}{d \xi} \frac{d \bar{c}}{d \xi}\right) d \xi \\
& =\frac{1}{2 \pi \sigma} \int_{\infty}^{-\infty}\left(-\frac{1}{\bar{\rho}} \frac{d^{2} \bar{c}}{d \xi^{2}}+\frac{1}{\bar{\rho}^{2}} \frac{d \bar{\rho}}{d \xi} \frac{d \bar{c}}{d \xi}\right) d \xi \\
& =\frac{1}{2 \pi \sigma} \int_{-\infty}^{\infty} \frac{d}{d \xi}\left(\frac{1}{\bar{\rho}} \frac{d \bar{c}}{d \xi}\right) d \xi=0
\end{aligned}
$$

and $z=z_{f}$. In the present work, the normalized distance $z=z_{f}$ was taken from Table 2 .
If the method is applied to process raw experimental data, then, the coordinate $z_{0}$ of the burning velocity surface may be calculated from the following equality:

$$
\bar{c}\left(z_{0}\right)=0.5
$$

using the measured profile of the Reynolds-averaged combustion progress variable. If the Favre-averaged combustion progress variable is measured, then the proper reference value may be calculated substituting $\bar{c}=0.5$ into (8). Under typical conditions, the reference value of $\tilde{c} \approx 0.1$ could be used (e.g., see bold solid line in Figure 6).

Third, turbulent burning velocity is considered to be equal to $\bar{w}_{e}\left(z_{f}\right)$ calculated from (14); see filled circle in Figure 6.

Results of the test, shown in Table 3, indicate that the values of $\bar{w}_{e}\left(z_{f}\right)$ (the second row) are very close to the normalized turbulent burning velocity $u_{t}$ calculated using (5) in all the studied flames, thus validating the considered method.

\section{Discussion}

From the physical standpoint, the above results could be interpreted as follows. Although the turbulent burning velocity $U_{t}$ is controlled by processes within (or, maybe, at the leading edge of) turbulent flame brush, $U_{t}$ can be evaluated by properly analyzing the velocity field in front of the flame brush, because the changes in this field, induced by the flame, are controlled by the burning velocity and the density ratio. For instance, in the simplest hypothetical case of an expanding spherical infinitely thin laminar flame, the velocity in unburned mixture is equal to $(\sigma-1) S_{L}\left(r / R_{f}\right)^{2}$, and the burning velocity can easily be evaluated by analyzing the radial velocity profile measured ahead of the flame.

The approximate equality of $u_{t}$ and $\left|\bar{w}_{e}\left(z_{f}\right)\right|$ may further be supported by invoking the so-called Zimont [3234] model of premixed turbulent combustion, extended, 


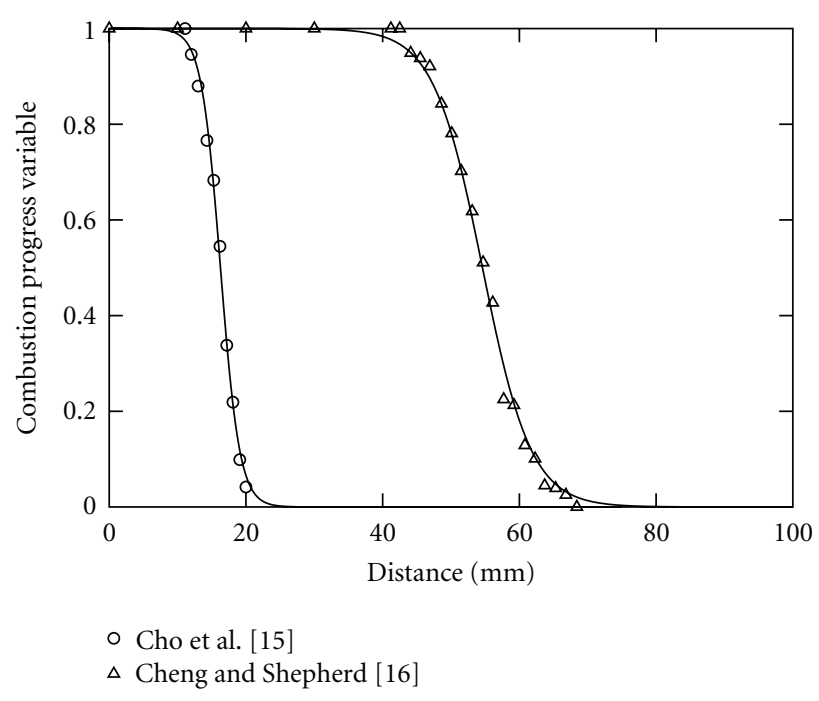

(a)

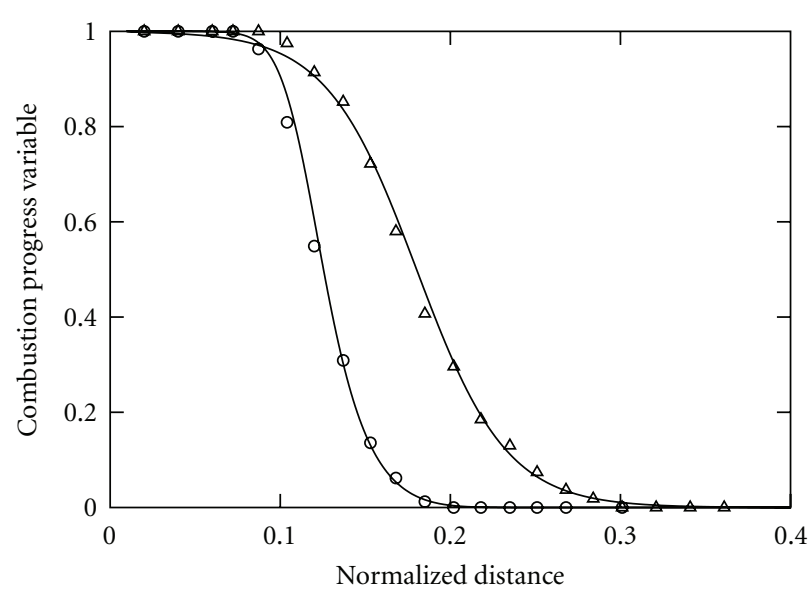

○ Li et al. [17], h4

$\Delta$ Li et al. [17], h6

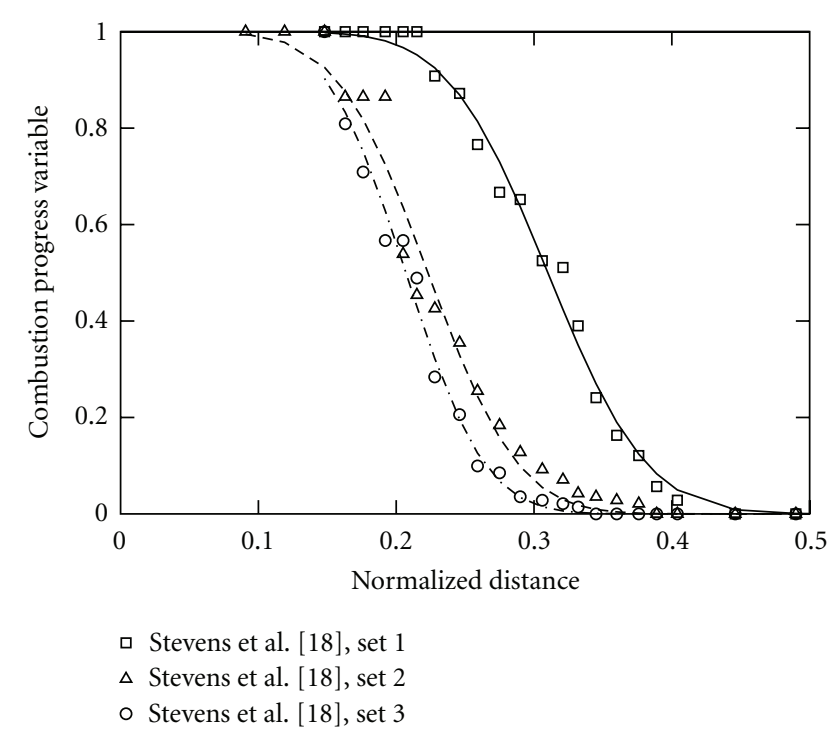

(b)

(c)

Figure 2: Axial profiles of (a) the Reynolds-averaged combustion progress variable $\bar{c}(x)$, measured by Cho et al. [15] and by Cheng and Shepherd [16], and the Favre-averaged combustion progress variable $\widetilde{c}(z)$, measured (b) by Li et al. [17] and (c) by Stevens et al. [18]. Symbols show experimental data. Curves have been obtained using (9).

and validated by the present author [35-37]. As reviewed elsewhere $[3,27]$, the model is successfully used by a number of different groups to simulate various turbulent flames.

The model deals with the following balance equation:

$$
\frac{\partial}{\partial t}(\bar{\rho} \tilde{c})+\frac{\partial}{\partial x_{j}}\left(\bar{\rho} \tilde{u}_{j} \tilde{c}\right)=\frac{\partial}{\partial x_{j}}\left(\bar{\rho} D_{t} \frac{\partial \tilde{c}}{\partial x_{j}}\right)+U_{t}|\nabla \tilde{c}|
$$

for the Favre-averaged combustion progress variable, where $D_{t}$ is turbulent diffusivity. Note that a basically similar balance equation was first introduced into the combustion literature by Prudnikov [38]. Along the axis of a statistically stationary flame stabilized in an impinging jet, this equation reads

$$
\bar{\rho} \widetilde{w} \frac{d \widetilde{c}}{d z}=d_{t} \frac{d}{d z}\left(\bar{\rho} \frac{d \widetilde{c}}{d z}\right)-u_{t} \frac{d \widetilde{c}}{d z}
$$

where $d_{t}=D_{t} /(U d)$ is the normalized turbulent diffusivity. Substitution of (8) and (9) into (20) yields

$$
\bar{\rho} \widetilde{w} \frac{d \widetilde{c}}{d z}=\frac{d_{t}}{\delta_{t}}\left(-2 \pi \xi \bar{\rho}-\frac{\sigma-1}{\sigma} \mathrm{e}^{-\pi \xi^{2}}\right) \frac{d \widetilde{c}}{d z}-u_{t} \frac{d \widetilde{c}}{d z} .
$$

Therefore, within the flame,

$$
\bar{\rho} \widetilde{w}=\frac{d_{t}}{\delta_{t}}\left(-2 \pi \xi \bar{\rho}-\frac{\sigma-1}{\sigma} \mathrm{e}^{-\pi \xi^{2}}\right)-u_{t} .
$$




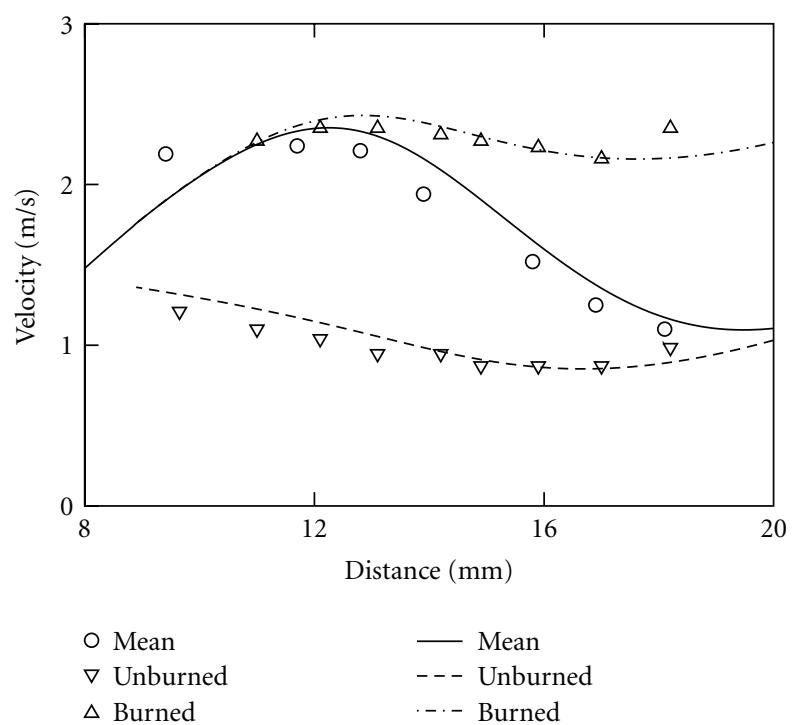

(a)

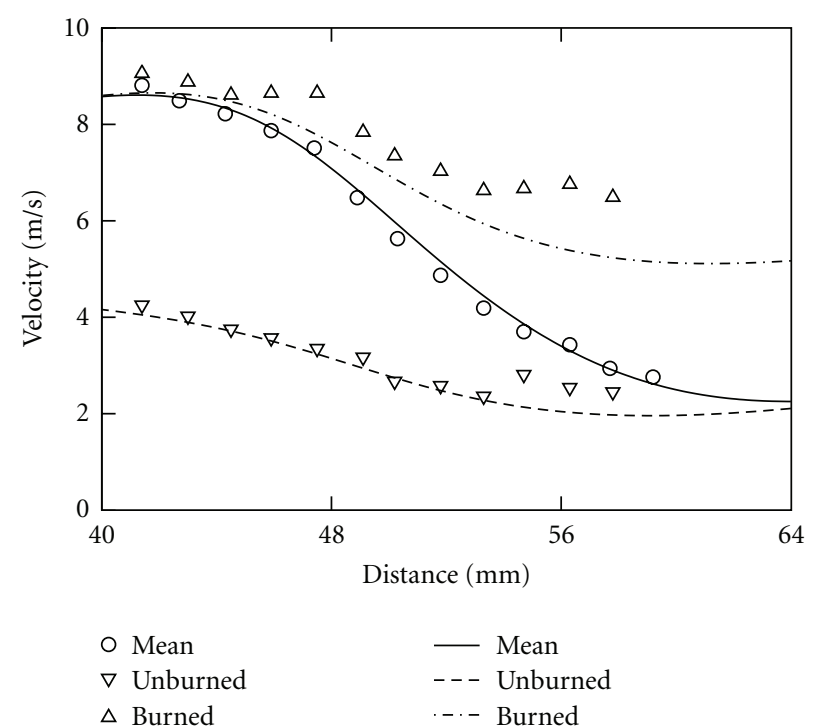

(b)

FIGURE 3: Axial profiles of the Reynolds-averaged and conditioned axial velocities in the flames investigated by Cho et al. [15] (a) and by Cheng and Shepherd [16] (b). Symbols show experimental data. Curves have been computed.

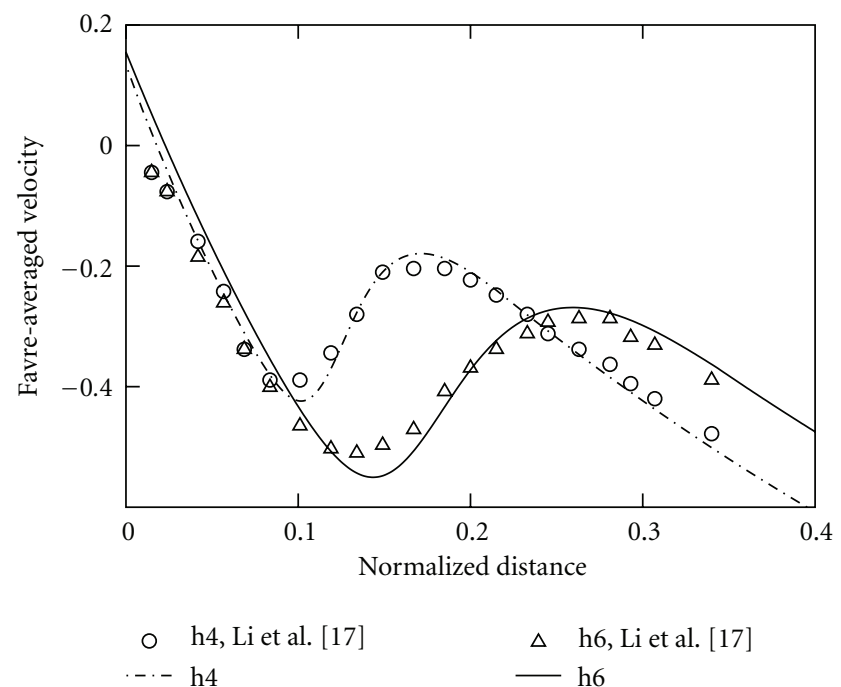

(a)

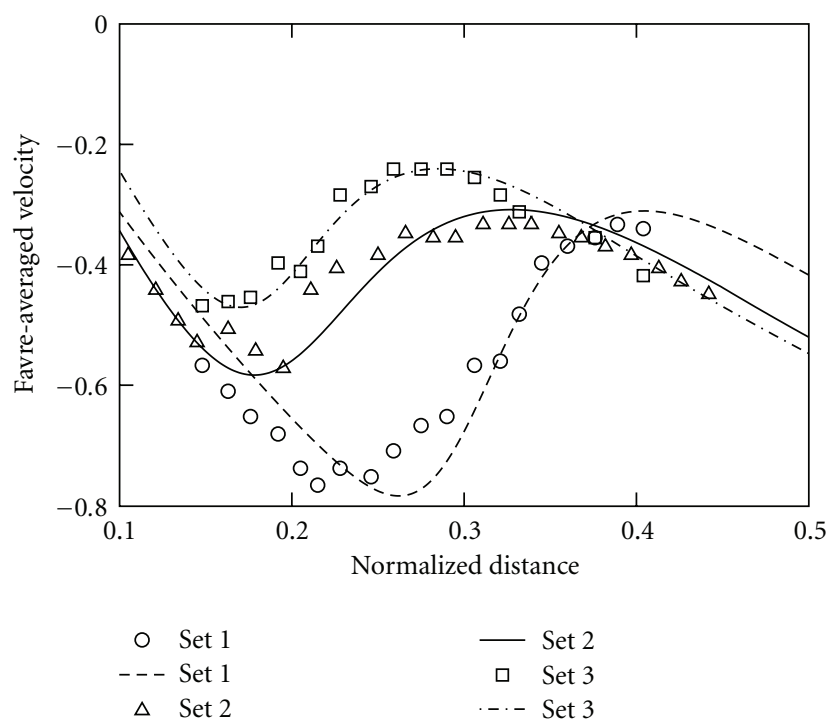

(b)

FIGURE 4: Axial profiles of the normalized Favre-averaged axial velocities $\widetilde{w}(z)$ in flames investigated by Li et al. [17] (a) and by Stevens et al. [18] (b). Symbols show experimental data. Curves have been computed.

TABLE 3: Turbulent burning velocities.

\begin{tabular}{lccccccc}
\hline Flame no. & 1 & 2 & 3 & 4 & 5 & 6 & 7 \\
\hline$u_{t}$ & 0.094 & 0.255 & 0.092 & 0.131 & 0.189 & 0.144 & 0.116 \\
$-\bar{w}_{e}\left(z_{f}\right)$ & 0.092 & 0.254 & 0.093 & 0.134 & 0.191 & 0.144 & 0.116 \\
$\left(g_{1}^{2}-Q\right)\left[z_{f}-z(\bar{c}=0.1)\right]^{2}$ & 0.002 & -0.0002 & 0.002 & 0.005 & 0.002 & 0.008 & 0.004 \\
$-\int_{0}^{1} 2 \bar{\rho} \widetilde{c}(1-\tilde{c})\left(\bar{g}_{b}-\bar{g}_{u}\right) d z$ & 0.006 & 0.004 & 0.004 & 0.009 & 0.004 & 0.014 & 0.009 \\
{$\left[2 \bar{\rho} \widetilde{c}(1-\tilde{c})\left(\bar{g}_{b}-\bar{g}_{u}\right) / \bar{\rho} \widetilde{w}(d \tilde{c} / d z)\right]_{\bar{c}=01}$} & 0.03 & 0.01 & 0.02 & 0.03 & 0.009 & 0.03 & 0.025 \\
\hline
\end{tabular}




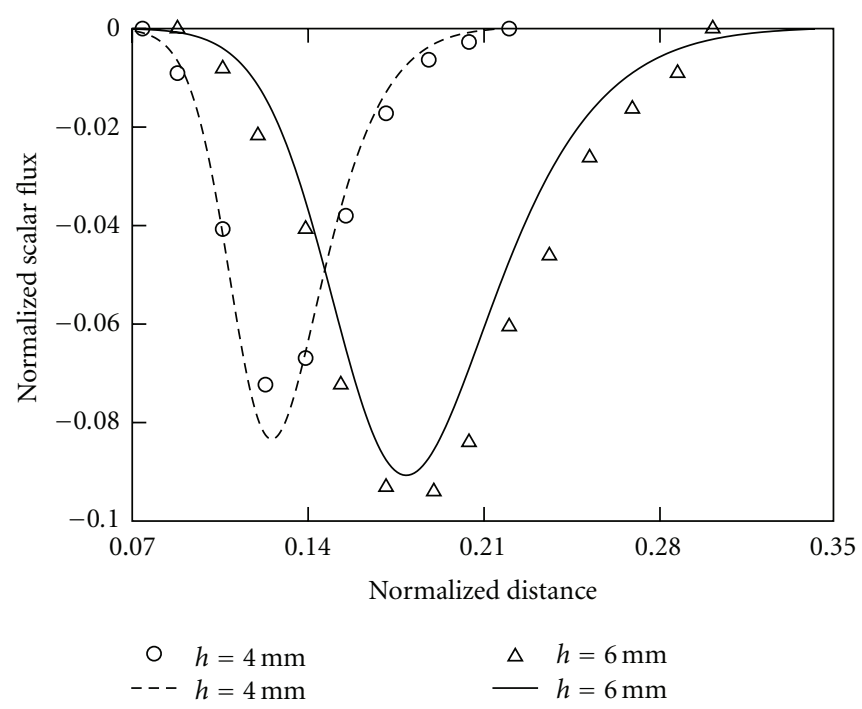

(a)

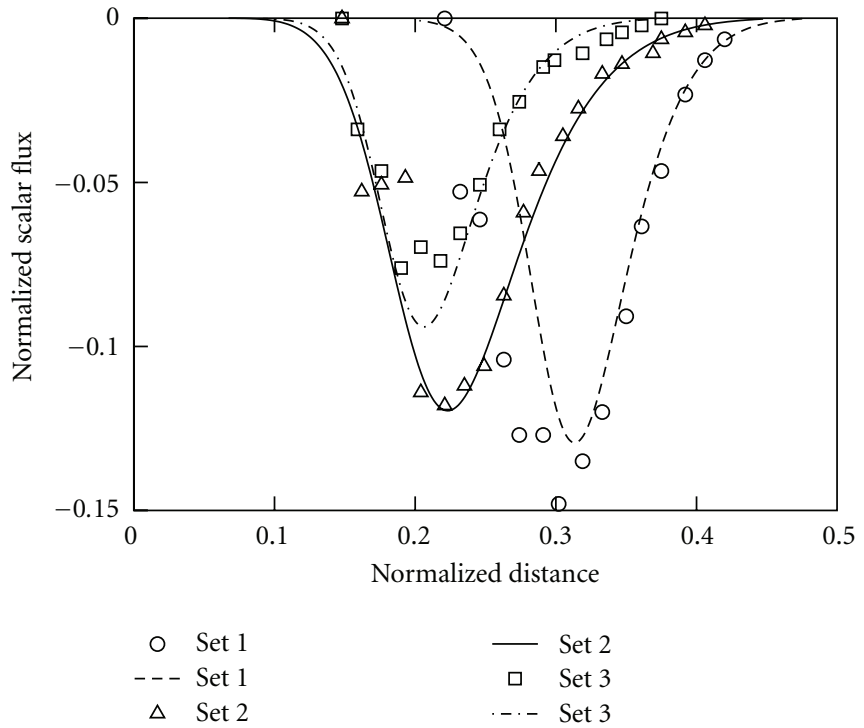

(b)

FIGURE 5: Axial profiles of the normalized axial turbulent scalar fluxes $\overline{\rho w^{\prime \prime} c^{\prime \prime}}(z)$ in flames investigated by Li et al. [17] (a) and by Stevens et al. [18] (b). Symbols show experimental data. Curves have been computed.

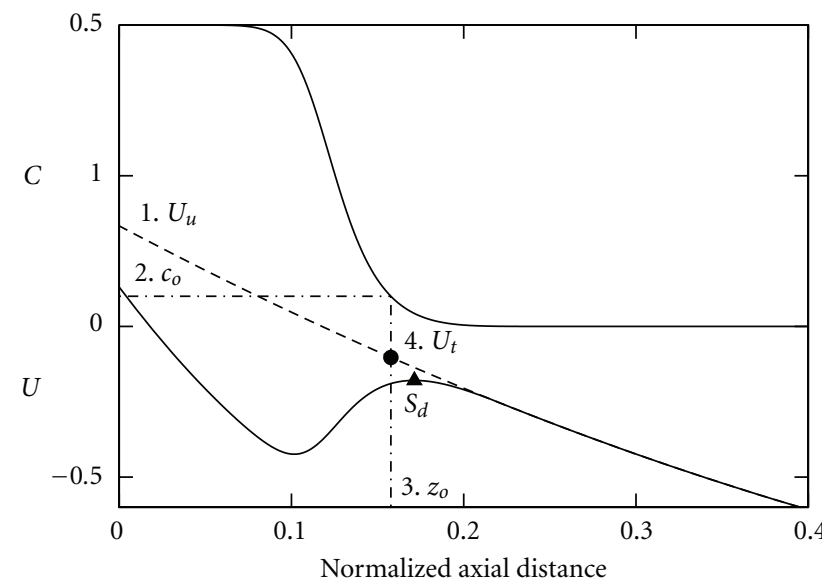

FIGURE 6: Tested method for determining turbulent burning velocity. Bold and thin solid lines show typical axial profiles of the Favre-averaged combustion progress variable and normalized axial velocity, respectively. Dashed curve calculated using (14) extrapolates the mean velocity profile from the oncoming flow to the flame brush. Filled circle shows turbulent burning velocity determined using the tested method. Filled triangle shows turbulent displacement speed associated commonly with the local minimum of $|\widetilde{w}(z)|$.

Differentiating this equation and using (8), (10), and (15), we obtain

$$
2 \bar{\rho} \tilde{g}=-\frac{1}{\delta_{t}} \frac{d \bar{\rho} \tilde{w}}{d \xi}=2 \pi \bar{\rho} \frac{d_{t}}{\delta_{t}^{2}} .
$$

Let us consider the leading edge of a turbulent flame brush and match (22) and (23), which are valid within the flame, with (14), which is valid in the unburned mixture.
Because $\bar{\rho} \rightarrow 1, \bar{c} \rightarrow 0$, and $\xi \gg 1$ at the leading edge $z \rightarrow z_{*}$, we obtain

$$
\begin{gathered}
-2 \pi d_{t} \frac{z_{*}-z_{f}}{\delta_{t}^{2}}-u_{t}=2 g_{1}\left(1-z_{*}\right)-\left(g_{1}^{2}-Q\right)\left(1-z_{*}\right)^{2}-1, \\
\pi \frac{d_{t}}{\delta_{t}^{2}}=g_{1}-\left(g_{1}^{2}-Q\right)\left(1-z_{*}\right) .
\end{gathered}
$$

Simple algebraic manipulations with these two equations and (14) result in

$$
u_{t}=-w_{e}\left(z_{f}\right)+\left(g_{1}^{2}-Q\right)\left(z_{f}-z_{*}\right)^{2} .
$$

According to the present simulations (see the third row in Table 3 and curves 1 in Figure 7), the last term in (25) is small at the leading edges of all the seven flames addressed in the present paper. Therefore, the model predicts that $u_{t} \approx-w_{e}\left(z_{f}\right)$ in a premixed turbulent flame stabilized in a circular impinging jet. Note that a similar analysis of a flame stabilized in a planar impinging jet yields exactly $u_{t}=$ $-w_{u}\left(z_{f}\right)$.

Certainly, the above substantiation of the studied method (i.e., $u_{t}=-\bar{w}_{e}\left(z_{f}\right)$ ) by invoking the extended Zimont model may be put into question by referring to a paper by Bray et al. [21] who claimed that the model failed in predicting the burning rate within flames stabilized in impinging jets, particularly, in flames nos. 2-5 studied in the present paper. More specifically, Bray et al. [21] have found that the following simple expression:

$$
\bar{W}=U_{t}|\nabla \tilde{c}|
$$

yields too strong dependence of $\bar{W}$ on $\tilde{c}$ if the turbulent burning velocity $U_{t}$ is evaluated using local Favre-averaged 


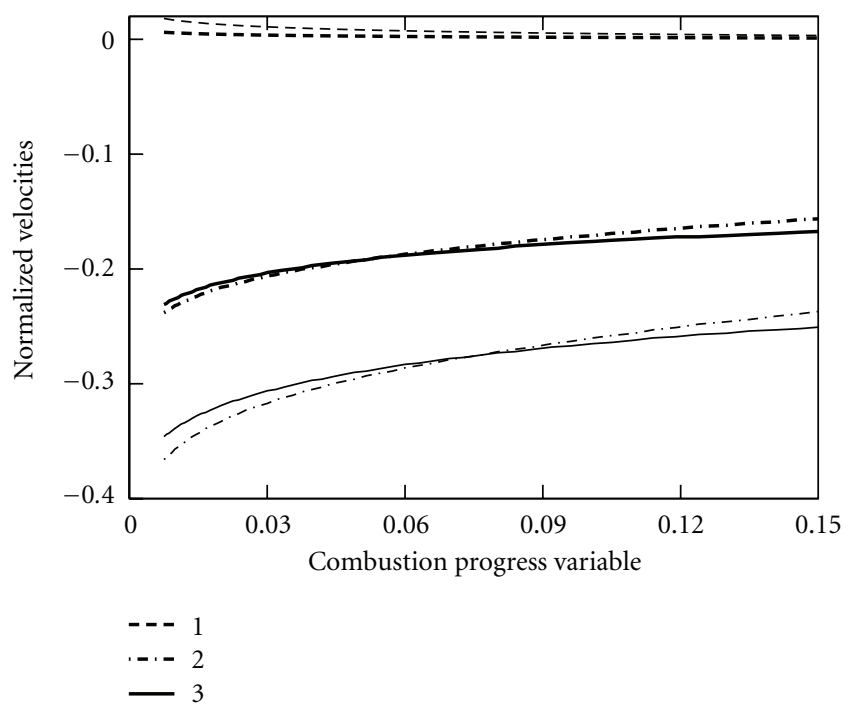

Figure 7: Term $\left(g_{1}^{2}-Q\right)\left(z_{f}-z\right)^{2}$ in (25) (1) and mean axial velocities calculated using (2) equation (14) and (3) equation (22) versus the Reynolds-averaged combustion progress variable. Results shown in bold and thin lines have been obtained for flames h4 and h6, respectively, investigated by Li et al. [17].

turbulence characteristics (turbulent kinetic energy $\tilde{k}$ and its dissipation rate $\widetilde{\mathcal{E}}$ ), which depend substantially on $\widetilde{c}$.

As pointed out elsewhere [39], the model tested by Bray et al. [21] differed substantially from the model developed and validated by Zimont and Lipatnikov [35-37]. In particular, the latter model provides a joint closure of the sum of the transport and reaction terms in the following well-known [13] balance equation:

$$
\frac{\partial}{\partial t}(\bar{\rho} \widetilde{c})+\frac{\partial}{\partial x_{k}}\left(\bar{\rho} \tilde{u}_{k} \widetilde{c}\right)=-\frac{\partial}{\partial x_{k}}\left(\overline{\rho u_{k}^{\prime \prime} c^{\prime \prime}}\right)+\bar{W}
$$

that is,

$$
-\frac{\partial}{\partial x_{j}} \overline{\rho u_{j}^{\prime \prime} c^{\prime \prime}}+\bar{W}=\frac{\partial}{\partial x_{j}}\left(\bar{\rho} D_{t} \frac{\partial \tilde{c}}{\partial x_{j}}\right)+U_{t}|\nabla \tilde{c}|,
$$

within the framework of the extended Zimont model. If applied to a flame stabilized in an impinging jet, (28) reads

$$
\begin{gathered}
\underbrace{-\frac{\partial}{\partial z}\left[\bar{\rho} \tilde{c}(1-\tilde{c})\left(\bar{w}_{b}-\bar{w}_{u}\right)\right]}_{1} \underbrace{-2 \bar{\rho} \tilde{c}(1-\tilde{c})\left(\bar{g}_{b}-\bar{g}_{u}\right)}_{2}+\underbrace{\Omega}_{3} \\
=\underbrace{d_{t} \frac{\partial}{\partial z}\left(\bar{\rho} \frac{\partial \tilde{c}}{\partial z}\right)}_{4}+\underbrace{u_{t}|\nabla \tilde{c}|}_{5} .
\end{gathered}
$$

Different terms in (29) are shown in Figure 8 for the flame h4 investigated by Li et al. [17], and similar results were obtained for the other six flames. In these calculations, the burning velocity used by the extended Zimont model was evaluated by integrating (29), that is,

$$
u_{t}=\int_{0}^{1} \Omega d z-\int_{0}^{1} 2 \bar{\rho} \widetilde{c}(1-\widetilde{c})\left(\bar{g}_{b}-\bar{g}_{u}\right) d z,
$$

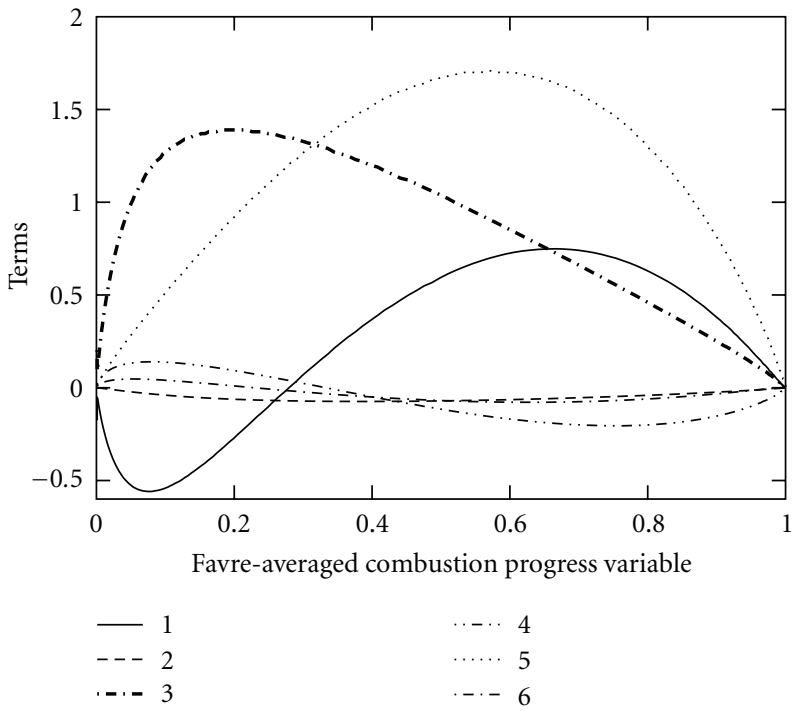

Figure 8: Different terms in (29), normalized using $d$ and $U$, versus the Favre-averaged combustion progress variable. Curves have been computed in the flame $\mathrm{h} 4$ investigated by $\mathrm{Li}$ et al. [17]. Term numbers are specified in legends. Curve 6 shows the difference between the LHS and RHS of (29).

and the normalized turbulent diffusivity was set in order to satisfy (29) at a point characterized by the maximum magnitude of the diffusion term 4 . Thus, neither $u_{t}$ nor $d_{t}$ depend on $z$. As discussed earlier [39], the use of a constant turbulent burning velocity within such flames appears to be more consistent with the underlying physics than substitution of $\tilde{k}(\tilde{c})$ into an expression for $U_{t}=U_{t}\left(S_{L}^{2} / \tilde{k}, \ldots\right)$, because the Favre-averaged $\tilde{k}$ strongly overestimates the turbulent kinetic energy due to the substantial contribution of the unburnedburned intermittency into $\tilde{k}$, for example, see Figure 1 in [39].

Figure 8 shows that although the source terms on the LHS and RHS of (29) depend differently on the mean combustion progress variable (compare with curves 3 and 5), (29) is sufficiently accurate in all the studied flames (see curve 6 which shows the difference between the LHS and RHS).

Moreover, (23), which results from the extended Zimont model, yields a constant radial gradient $\tilde{g}$ of the Favreaveraged radial velocity across a premixed turbulent flame stabilized in an impinging jet provided that $u_{t}=$ const and $d_{t}=$ const. Numerical results plotted in Figure 9 show that $\tilde{g}$ is approximately constant in the range $\bar{c}<0.8$ of turbulent flame brush, thus further validating the model in the largest part of turbulent flame brush.

Furthermore, curves 2 and 3 in Figure 7 show velocities calculated using (14) and (22), respectively, at the leading edges of the two flames investigated by $\mathrm{Li}$ et al. [17]. The latter curves have been calculated by neglecting term proportional to $\mathrm{e}^{-\pi \xi^{2}}$, as it vanishes at the leading edge. The fact that curves 2 and 3 are very close to one another further supports the extended Zimont model. Similar results were computed for other studied flames. 


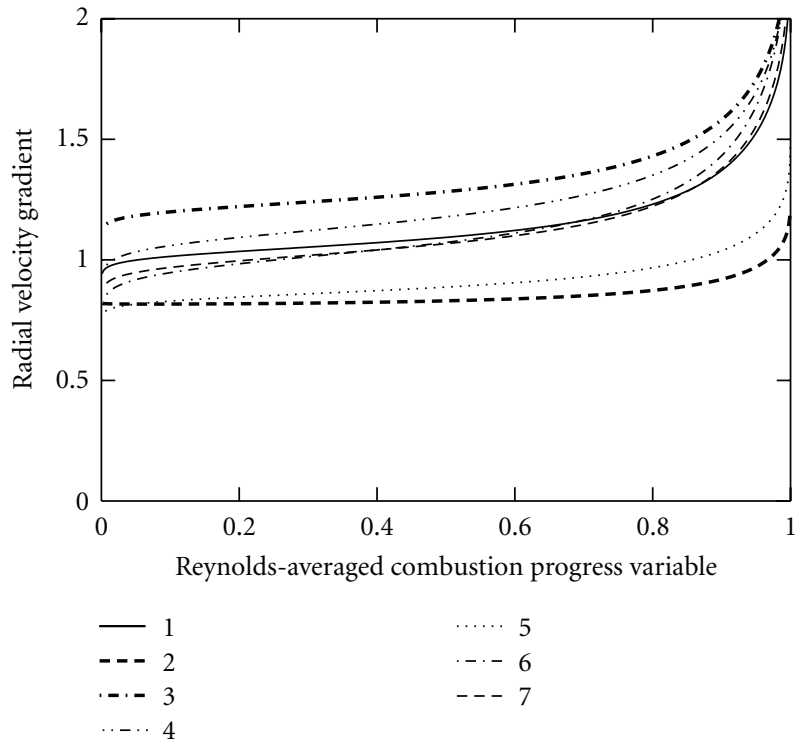

FIGURE 9: Normalized radial gradient of the Favre-averaged radial velocity versus the Reynolds-averaged combustion progress variable. Numbers in legends correspond to flame numbers in Table 1.

Thus, the extended Zimont model is consistent with the experimental data obtained from premixed turbulent flames stabilized in impinging jets and, therefore, may be used to substantiate the tested method for evaluating turbulent burning velocity in such flames, as done above (Although the model was also put into question by Peters [40], his arguments were rebutted in earlier papers [28,29].) .

Finally, it is worth raising the following issue. To the the best of the present author's knowledge, the key equation of the extended Zimont model, that is, (19), has yet been substantiated by basic arguments $[3,28,29,32,34,38]$ only in the case of a statistically planar one-dimensional premixed turbulent flame. Therefore, strictly speaking, the extended Zimont model yields the following joint closure:

$$
-\frac{\partial}{\partial \xi} \overline{\rho u_{n}^{\prime \prime} c^{\prime \prime}}+\bar{W}=\frac{\partial}{\partial \xi}\left(\bar{\rho} D_{t} \frac{\partial \widetilde{c}}{\partial \xi}\right)+U_{t}|\nabla \tilde{c}|,
$$

where the $\xi$-axis is locally normal to the mean flame brush and $u_{n}$ is the flow velocity in this direction. Accordingly, the model may be considered to avoid closing the turbulent scalar flux in the tangential direction, and the following balance equation:

$$
\underbrace{-\frac{\partial}{\partial z}\left[\bar{\rho} \widetilde{c}(1-\tilde{c})\left(\bar{w}_{b}-\bar{w}_{u}\right)\right]}_{\mathrm{I}}+\underbrace{\Omega}_{\mathrm{III}}=\underbrace{d_{t} \frac{\partial}{\partial z}\left(\bar{\rho} \frac{\partial \tilde{c}}{\partial z}\right)}_{\mathrm{IV}}+\underbrace{u_{t}|\nabla \tilde{c}|}_{\mathrm{V}}
$$

may be applied to a flame stabilized in an impinging jet by referring to the same extended Zimont model. While (29) invokes this model and a gradient closure of the transverse turbulent scalar flux (the closure terms vanishes along the jet axis for symmetry reasons), (32) invokes the same model and the BML (7) in order to close the transverse flux (the corresponding terms are not shown on the LHS and RHS of (32), because they cancel one another).

Terms I and III on the LHS of (32) are equal to terms 1 and 3, respectively, on the LHS of (29), but the former equation does not involve a counterpart to term 2, which is much smaller than terms 1 and 5 (see Figure 8). Our simulations show that terms IV and V on the RHS of (32) are close to terms 4 and 5, respectively, on the RHS of (29), with the difference between the two sets of terms resulting from different $d_{t}$ and $u_{t}$ substituted into the two equations. Indeed, integration of (32) leads us to (5), with the difference between it and (30) consisting of the second term on the RHS of the latter equation. Comparison of the first and fourth rows in Table 3 shows that the integrated transverse scalar flux is much less than the integrated heat release rate in all the seven flames addressed in the present paper, with the largest difference $(10 \%)$ being computed in flame no. 6 (set 2 investigated by Stevens et al. [18]). Thus, Figure 8 qualitatively describes the behavior of both terms $1-5$ in (29) and terms I-V in (32) in all the flames studied here, but term II vanishes in the latter equation.

If (29) is substituted with (32), then (21) should also be substituted with the following equation:

$$
\begin{aligned}
\bar{\rho} \widetilde{w} \frac{d \widetilde{c}}{d z} & +2 \widetilde{\rho} \widetilde{c}(1-\widetilde{c})\left(\bar{g}_{b}-\bar{g}_{u}\right) \\
& =\frac{d_{t}}{\delta_{t}}\left(-2 \pi \xi \bar{\rho}-\frac{\sigma-1}{\sigma} \mathrm{e}^{-\pi \xi^{2}}\right) \frac{d \widetilde{c}}{d z}-u_{t} \frac{d \widetilde{c}}{d z},
\end{aligned}
$$

that is, the second term is introduced into the LHS. Accordingly, (22)-(25), which result from (21), do not result from (33). However, because the introduced term is much less than the mean convection term at the leading edges of all the seven flames simulated here (see the fifth row in Table 3 ), (25) appears to be sufficiently accurate even if (32) and (33) are invoked. At the same time, the variations in $\tilde{g}$ within the studied flames (see Figure 9) may in part be caused by the transverse flux introduced into (33), as well as by the eventual dependencies of $u_{t}$ and $d_{t}$ on $z$ due to variations in turbulence characteristics within the flames.

\section{Conclusions}

A method for evaluating burning velocity in premixed turbulent flames stabilized in divergent mean flows, proposed earlier by Lipatnikov and Chomiak [10], was quantitatively validated using numerical approximations of measured axial profiles of the mean combustion progress variable, mean and conditioned axial velocities, and axial turbulent scalar flux, obtained by Cho et al. [15], Cheng and Shepherd [16], Li et al. [17], and Stevens et al. [18] from seven different flames each stabilized in an impinging jet.

The method is further substantiated by analyzing the combustion progress variable balance equation that is yielded by the extended Zimont model of premixed turbulent combustion. The compatibility of the model with the aforementioned experimental data is also shown. 


\section{Acknowledgments}

The author would like to express his sincere thanks to Professors Jerzy Chomiak and Vladimir Sabel'nikov for valuable discussions. This work was supported by the Swedish Research Council (VR), Swedish Energy Agency, and by the Combustion Engine Research Center (CERC).

\section{References}

[1] Ö. L. Gülder, "Turbulent premixed flame propagation models for different combustion regimes," Proceedings of the Combustion Institute, vol. 23, pp. 743-750, 1990.

[2] D. Bradley, "How fast can we burn?" Proceedings of the Combustion Institute, vol. 24, pp. 247-262, 1992.

[3] A. N. Lipatnikov and J. Chomiak, "Turbulent flame speed and thickness: phenomenology, evaluation, and application in multi-dimensional simulations," Progress in Energy and Combustion Science, vol. 28, no. 1, pp. 1-74, 2002.

[4] J. F. Driscoll, "Turbulent premixed combustion: flamelet structure and its effect on turbulent burning velocities," Progress in Energy and Combustion Science, vol. 34, no. 1, pp. 91-134, 2008.

[5] I. G. Shepherd and L. W. Kostiuk, "The burning rate of premixed turbulent flames in divergent flows," Combustion and Flame, vol. 96, no. 4, pp. 371-380, 1994.

[6] F. C. Gouldin, "Combustion intensity and burning rate integral of premixed flames," Proceedings of the Combustion Institute, vol. 26, no. 1, pp. 381-388, 1996.

[7] A. N. Lipatnikov and J. Chomiak, "Effects of premixed flames on turbulence and turbulent scalar transport," Progress in Energy and Combustion Science, vol. 36, no. 1, pp. 1-102, 2010.

[8] P. Clavin, "Dynamical behavior of premixed flame fronts in laminar and turbulent flows," Progress in Energy and Combustion Science, vol. 11, no. 1, pp. 1-59, 1985.

[9] A. N. Lipatnikov and J. Chomiak, "Global stretch effects in premixed turbulent combustion," Proceedings of the Combustion Institute, vol. 31, pp. 1361-1368, 2007.

[10] A. N. Lipatnikov and J. Chomiak, "Turbulent burning velocity and speed of developing, curved, and strained flames," Proceedings of the Combustion Institute, vol. 29, pp. 2113-2121, 2002.

[11] D. Bradley, M. Z. Haq, R. A. Hicks et al., “Turbulent burning velocity, burned gas distribution, and associated flame surface definition," Combustion and Flame, vol. 133, no. 4, pp. 415430, 2003.

[12] A. N. Lipatnikov and J. Chomiak, "Comment on "Turbulent burning velocity, burned gas distribution, and associated flame surface definition": D. Bradley, M. Z. Haq, R. A. Hicks, T. Kitagawa, M. Lawes, C. G. W. Sheppard, R. Woolley, Combust. Flame 133 (2003) 415," Combustion and Flame, vol. 137, no. 12, pp. 261-263, 2004.

[13] K. N. C. Bray and J. B. Moss, "A unified statistical model of the premixed turbulent flame," Acta Astronautica, vol. 4, no. 3-4, pp. 291-319, 1977.

[14] A. N. Lipatimikov and J. Chomiak, "Numerical tests of a measurement method for turbulent burning velocity in stagnation flames," Combustion Science and Technology, vol. 178, no. 6, pp. 1117-1141, 2006.

[15] P. Cho, C. K. Law, R. K. Cheng, and I. G. Shepherd, "Velocity and scalar fields of turbulent premixed flames in stagnation flow," Proceedings of the Combustion Institute, vol. 22, no. 1, pp. 739-745, 1988.
[16] R. K. Cheng and I. G. Shepherd, "The influence of burner geometry on premixed turbulent flame propagation," Combustion and Flame, vol. 85, no. 1-2, pp. 7-26, 1991.

[17] S. C. Li, P. A. Libby, and F. A. Williams, "Experimental investigation of a premixed flame in an impinging turbulent stream," Proceedings of the Combustion Institute, vol. 25, pp. 1207-1214, 1994.

[18] E. J. Stevens, K. N. C. Bray, and B. Lecordier, "Velocity and scalar statistics for premixed turbulent stagnation flames using PIV," Proceedings of the Combustion Institute, vol. 1, pp. 949955, 1998.

[19] K. N. C. Bray, M. Champion, and P. A. Libby, "Premixed flames in stagnating turbulence part II : the mean velocities and pressure and the Damköhler number," Combustion and Flame, vol. 112, no. 4, pp. 635-654, 1998.

[20] K. N. C. Bray, M. Champion, and P. A. Libby, "Premixed flames in stagnating turbulence Part IV: a new theory for the Reynolds stresses and Reynolds fluxes applied to impinging flows," Combustion and Flame, vol. 120, no. 1-2, pp. 1-18, 2000.

[21] K. N. C. Bray, M. Champion, and P. A. Libby, "Premixed flames in stagnating turbulence Part V-evaluation of models for the chemical source term," Combustion and Flame, vol. 127, no. 1-2, pp. 2023-2040, 2001.

[22] F. Biagioli and V. L. Zimont, "Gasdynamics modelling of counter-gradient transport in open and impinging turbulent premixed flames," Proceedings of the Combustion Institute, vol. 29, pp. 2087-2095, 2002.

[23] F. Biagioli, "Position, thickness and transport properties of turbulent premixed flames in stagnating flows," Combustion Theory and Modelling, vol. 8, no. 3, pp. 533-554, 2004.

[24] A. N. Lipatnikov, "A test of conditioned balance equation approach," Proceedings of the Combustion Institute, vol. 33, pp. 1497-1504, 2011.

[25] P. A. Libby and K. N. C. Bray, "Variable density effects in premixed turbulent flames," AIAA Journal, vol. 15, no. 8, pp. 1186-1193, 1977.

[26] K. J. Bosschaart and L. P. H. De Goey, "The laminar burning velocity of flames propagating in mixtures of hydrocarbons and air measured with the heat flux method," Combustion and Flame, vol. 136, no. 3, pp. 261-269, 2004.

[27] A. N. Lipatnikov, "Premixed turbulent flame as a developing front with a self-similar structure," in Focus on Combustion Research, S. Z. Jiang, Ed., chapter 3, pp. 89-141, Nova Science Publishers, New York, NY, USA, 2006.

[28] A. N. Lipatnikov and J. Chomiak, "A theoretical study of premixed turbulent flame development," Proceedings of the Combustion Institute, vol. 30, pp. 843-850, 2005.

[29] A. N. Lipatnikov and J. Chomiak, "Self-similarly developing, premixed, turbulent flames: a theoretical study," Physics of Fluids, vol. 17, no. 6, Article ID 065105, 15 pages, 2005.

[30] A. N. Lipatnikov, "Conditionally averaged balance equations for modeling premixed turbulent combustion in flamelet regime," Combustion and Flame, vol. 152, no. 4, pp. 529-547, 2008.

[31] A. N. Lipatnikov, "Transient behavior of turbulent scalar transport in premixed flames," Flow, Turbulence and Combustion, vol. 86, no. 3-4, pp. 609-637, 2011.

[32] V. L. Zimont, "To computations of turbulent combustion of partially premixed gases," in Chemical Physics of Combustion and Explosion Processes. Combustion of Multi-Phase and Gas Systems, pp. 77-80, OIKhF, Chernogolovka, Russia, 1977. 
[33] V. L. Zimont, "Theory of turbulent combustion of a homogeneous fuel mixture at high Reynolds numbers," Combustion, Explosion, and Shock Waves, vol. 15, no. 3, pp. 305-311, 1979.

[34] V. L. Zimont, "Gas premixed combustion at high turbulence. Turbulent flame closure combustion model," Experimental Thermal and Fluid Science, vol. 21, no. 1-3, pp. 179-186, 2000.

[35] V. L. Zimont and A. N. Lipatnikov, "To computations of the heat release rate in turbulent flames," Doklady Physical Chemistry, vol. 332, pp. 592-594, 1993.

[36] V. L. Zimont and A. N. Lipatnikov, "A numerical model of premixed turbulent combustion," Chemical Physics Reports, vol. 14, pp. 993-1025, 1995.

[37] V. P. Karpov, A. N. Lipatnikov, and V. L. Zimont, "A test of an engineering model of premixed turbulent combustion," Proceedings of the Combustion Institute, vol. 26, pp. 249-257, 1996.

[38] A. G. Prudnikov, "Burning of homogeneous fuel-air mixtures in a turbulent flow," in Physical Principles of the Working Process in Combustion Chambers of Jet Engines, B. V. Raushenbakh, Ed., chapter 5, pp. 244-236, Clearing House for Federal Scientific \& Technical Information, Springfield, Ohio, USA, 1967.

[39] A. N. Lipatnikov, "Comments on "Premixed flames in stagnating turbulence part V-Evaluation of models for the chemical source term" by K. N. C. Bray, M. Champion, and P. A. Libby," Combustion and Flame, vol. 131, no. 1-2, pp. 219-221, 2002.

[40] N. Peters, Turbulent Combustion, Cambridge University Press, Cambridge, 2000. 

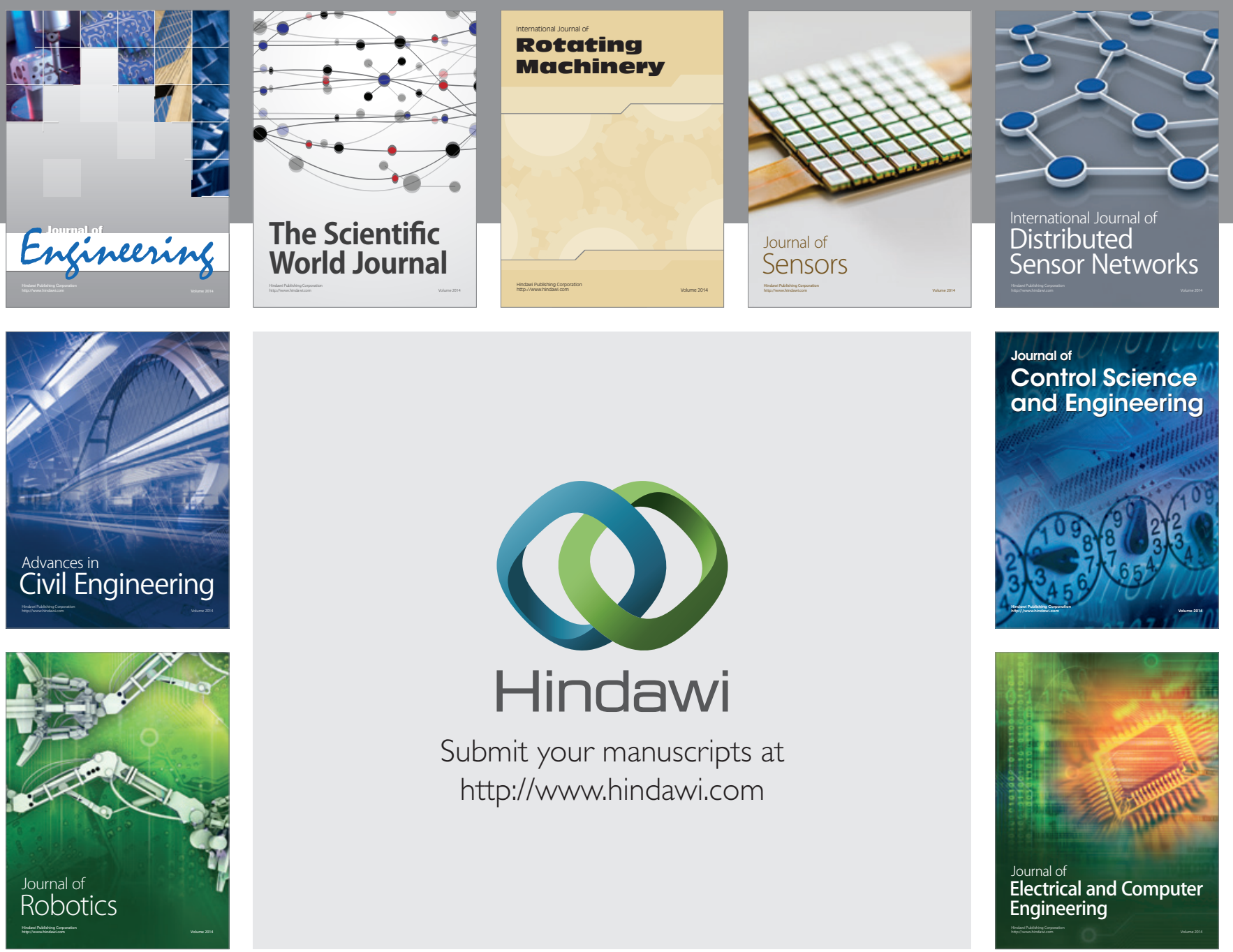

Submit your manuscripts at

http://www.hindawi.com
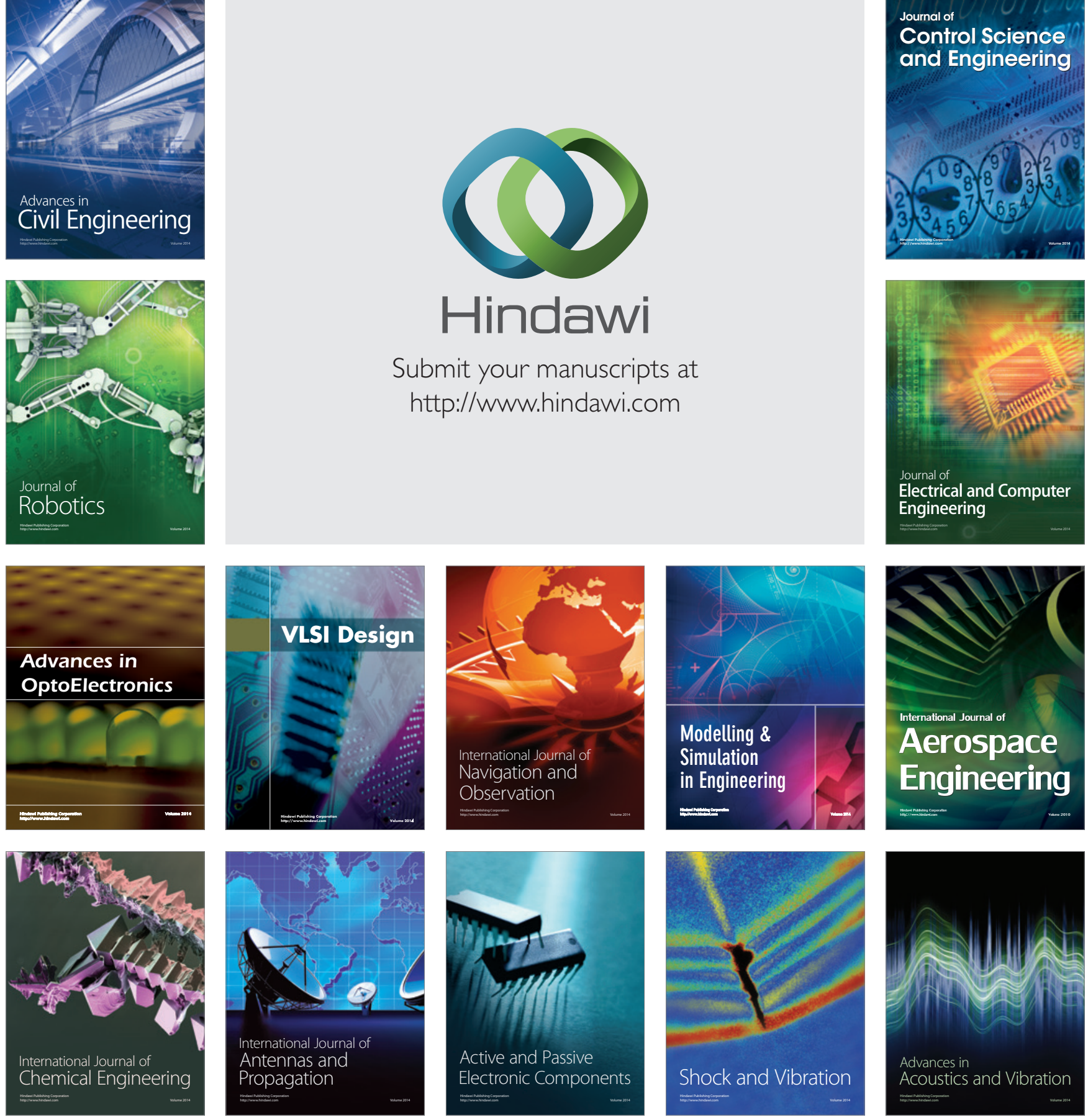